\title{
A tail like no other
}

\section{The RPC-MAG view of Rosetta's tail excursion at comet 67P/Churyumov-Gerasimenko}

\author{
Martin Volwerk ${ }^{1}$, Charlotte Goetz ${ }^{2}$, Ingo Richter ${ }^{2}$, Magda Delva ${ }^{1}$, Katharina Ostaszewski ${ }^{2}$, \\ Konrad Schwingenschuh ${ }^{1}$, and Karl-Heinz Glassmeier ${ }^{2}$ \\ 1 Space Research Institute, Austrian Academy of Sciences, Schmiedlstr. 6, 8042 Graz, Austria \\ e-mail: martin.volwerk@oeaw.ac.at \\ ${ }^{2}$ Institute for Geophysics and Extraterrestrial Physics, Technische Universtität Braunschweig, Mendelssohnstrasse 3,
} Braunschweig 38106, Germany

Received 30 October 2017 / Accepted 6 December 2017

\begin{abstract}
Context. The Rosetta Plasma Consortium (RPC) magnetometer (MAG) data during the tail excursion in March-April 2016 are used to investigate the magnetic structure of and activity in the tail region of the weakly outgassing comet $67 \mathrm{P} / \mathrm{Chury} u m o v-\mathrm{Gerasimenko}$ $(67 \mathrm{P})$.

Aims. The goal of this study is to compare the large scale (near) tail structure with that of earlier missions to strong outgassing comets, and the small scale turbulent energy cascade (un)related to the singing comet phenomenon.

Methods. The usual methods of space plasma physics are used to analyse the magnetometer data, such as minimum variance analysis, spectral analysis, and power law fitting. Also the cone angle and clock angle of the magnetic field are calculated to interpret the data. Results. It is found that comet 67P does not have a classical draped magnetic field and no bi-lobal tail structure at this late stage of the mission when the comet is already at 2.7 AU distance from the Sun. The main magnetic field direction seems to be more across the tail direction, which may implicate an asymmetric pick-up cloud. During periods of singing comet activity the propagation direction of the waves is at large angles with respect to the magnetic field and to the radial direction towards the comet. Turbulent cascade of magnetic energy from large to small scales is different in the presence of singing as without it.
\end{abstract}

Key words. plasmas - comets: individual: 67P/Churyumov-Gerasimanko

\section{Introduction}

The Rosetta mission (Glassmeier et al. 2007a) to comet 67P/Churyumov-Gerasimenko (67P) was equipped with the Rosetta Plasma Consortium package (RPC; Carr et al. 2007) to investigate the plasma environment of the comet and its interaction with the solar wind. Because of various constraints during the mission, most observations were carried out within a radial distance of $\sim 100 \mathrm{~km}$ from the nucleus. A full discussion of the magnetic field around comet $67 \mathrm{P}$ is provided in Goetz et al. (2017). However, from 22 September to 11 October 2015, the socalled dayside excursion took place, during which Rosetta moved $\sim 1500 \mathrm{~km}$ upstream of the comet. It was envisioned to encounter the bow shock of the comet, which unfortunately was not found (see Edberg et al. 2016; Mandt et al. 2016) as the excursion stopped too close to the comet owing to operational constraints.

The most prominent features of a comet, as observed from the ground, are its ion and dust tails. So far only one fly-by of a comet happened to cross a comet's tail: the ICE satellite sampled comet 27P/Giacobini-Zinner's tail on 11 September 1985 (see e.g. Slavin et al. 1986). It was shown that the tail consisted of a bilobed magnetic field structure as one would expect from magnetic field line draping around the active cometary nucleus that was described by Alfvén (1957).
However, comets like 27P/Giacobini-Zinner or 1P/Halley had strongly outgassing nuclei, whereas the Rosetta target $67 \mathrm{P}$ was only weakly outgassing with a maximum rate about two orders of magnitude lower than the active targets and a production rate of $10^{26} \mathrm{~s}^{-1}$ (Hansen et al. 2016) at perihelion; for the tail excursion discussed in this paper this value was lower again by about a factor $10, Q \sim 10^{25} \mathrm{~s}^{-1}$. This means that the solar wind-comet interaction is greatly reduced through a great reduction of ion pick-up. Indeed, Koenders et al. (2016) have already shown through simulations and data analysis that the behaviour of the magnetic field at perihelion does not follow the normal draping in the equatorial $X Y$-plane, but through deflection of the solar wind by the ion pick-up draping is mainly seen in the plane orthogonal to the equatorial plane (see Fig. 5 in Koenders et al. 2016). The development of the magnetic field during the whole Rosetta mission at comet $67 \mathrm{P}$ was discussed by Goetz et al. (2017), who found near-classical draping patterns around perihelion, but with a wide opening angle of the tail.

It was only decided near the end of the Rosetta mission in 2016 that a "tail excursion" would take place in March-April, when comet $67 \mathrm{P}$ was at a distance of $\sim 2.7$ astronomical units (AU) from the Sun. This heliocentric distance means that the activity of the comet has already decreased significantly, which may or may not have an effect on the creation of the cometary 


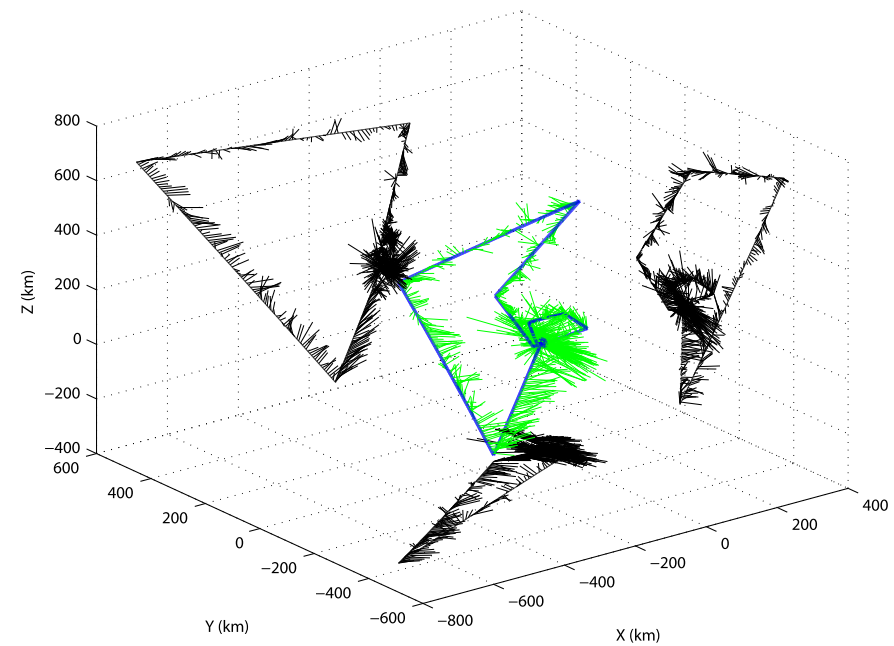

Fig. 1. Three-dimensional view of the magnetic field (green, one vector per hour) along orbit of Rosetta (blue) in the CSEQ coordinate system. The black parts are projections onto the three different planes.

tail. In all, the tail excursion follows a complicated path of four legs such that various regions, for example the ion and dust tail, is crossed. The orbit can be seen in Fig. 1 .

During the tail excursion the magnetic field strength around comet $67 \mathrm{P}$ was $B \approx 10 \mathrm{nT}$; this means that when water ions are picked up by the magnetic field of the solar wind at $v \approx$ $350 \mathrm{~km} \mathrm{~s}^{-1}$, the gyro radius of these ions is $\rho_{\mathrm{i}} \approx 6000 \mathrm{~km}$. This radius is much larger than the radial distance covered by Rosetta from the nucleus, and thus kinetic effects on the structure of the tail should be expected and not the typical MHD-type of tail structure.

In this paper first the large structure aspects are discussed, such as the presence of field line draping and the magnetic field strength around the comet as a function of radial distance. Then the small scale physics is discussed, specifically the singing comet and the turbulent cascade of wave energy around the comet (Richter et al. 2015, 2016).

For the study of the tail excursion in this paper only the Rosetta RPC magnetometer data (Glassmeier et al. 2007b) are used. The data have been processed and calibrated (see Goetz et al. 2016b) and are presented in the CSEQ ${ }^{1}$ coordinate system unless otherwise stated in the text.

\section{Near tail - the larger structure}

The Rosetta tail excursion took place in from 24 March until 10 April 2016, which is the only time that Rosetta spent a significant time at the nightside of comet 67P. In Fig. 2 the magnetometer data are shown; these data include derived quantities as clock and cone angle, the temperature of the sensor and the propagated solar wind (Tao et al. 2005), and the clock angle of the solar wind from OMNI data shifted by six days (derived from the ballistic propagation model Opitz et al. 2009, 2010), over a more extended period of 19 March until 19 April 2016. The magnetic field data are box-car averages of 512 second intervals shifted by $30 \mathrm{~s}$.

\footnotetext{
1 Original definition from the SPICE kernel: $+X$ axis is the position of the Sun relative to the body; it is the primary vector and points from the body to the Sun; $+Z$ axis is the component towards the north pole of the Sun of date orthogonal to the $+X$ axis; $+Y$ axis completes the righthanded reference frame; the origin of this frame is the centre of mass of the body (Acton 1996).
}

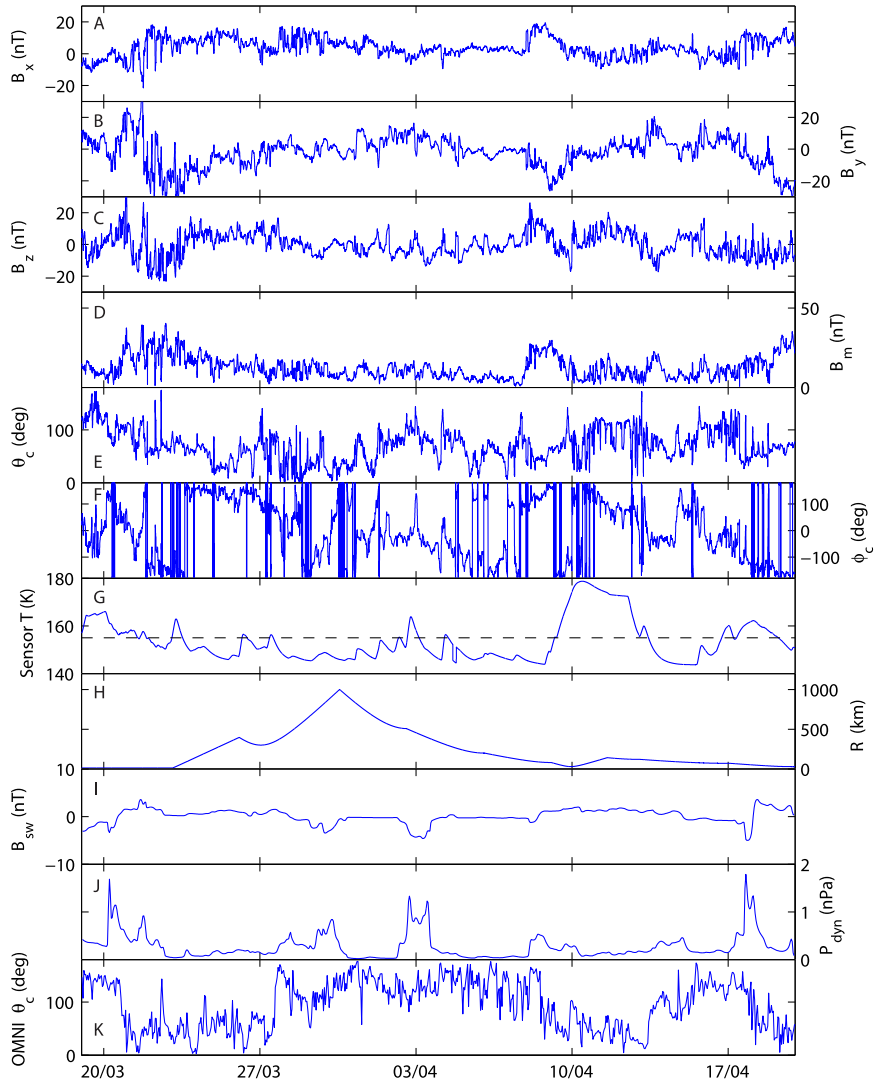

Fig. 2. Overview of the RPC magnetic field data and auxiliary data for the time interval 19 March to 19 April 2016, which are used to study the tail excursion. $(A-D)$ RPC MAG data $B_{x}, B_{y}, B_{z}, B_{\mathrm{m}} ;(E)$ and $(F)$ the cone-angle $\theta_{\mathrm{c}}$ and clock-angle $\phi_{\mathrm{c}}$ of the measured magnetic field; $(G)$ sensor temperature; $(H)$ radial distance $R$ of Rosetta to comet 67P; $(I)$ and $(J)$ propagated solar wind tangential magnetic field $B_{\mathrm{sw}}$ and dynamic pressure $P_{\text {dyn }}$ using Tao et al. (2005); and $(K)$ clock angle of the solar wind magnetic field from OMNI shifted by 6 days.

In Fig. 2G the sensor temperature of the magnetometer is shown, which is necessary for the calibration of the MAG data. The instrument has a temperature dependent offset and is measuring in a magnetically non-clean environment (Goetz et al. $2016 b$ ). Using the observations of the diamagnetic cavity around comet 67P (Goetz et al. 2017), a new temperature dependent model could be found for the period April 2015 to February 2016. This model is officially only valid for a sensor temperature range $155 \leq T_{\mathrm{s}} \leq 210 \mathrm{~K}$. It is clear from Fig. $2 \mathrm{G}$ that part of the tail excursion was measured with $T_{\mathrm{s}}<155 \mathrm{~K}$, and thus the calibration could be imperfect. However, to exclude the intervals for which $T_{\mathrm{s}}<155 \mathrm{~K}$ would reduce the available data to an unworkable dearth along the tail excursion. Therefore, all data are used, but in case of peculiar results the calibration problem should be kept in mind, and these results are addressed in the discussion section of this paper.

\subsection{Field line draping}

Starting at 24 March, Rosetta moves away from comet 67P up to a radial distance $R$ of $\sim 1000 \mathrm{~km}$ (Fig. 2G). Assuming that the solar wind-comet interaction is simply described by the Alfvén draping model (Alfvén 1957) and Parker-spiral solar wind, then one would expect a change in the cone angle of the magnetic field when Rosetta crosses the centre of the tail. 
The cone angle, defined as

$\theta_{\mathrm{c}}=\tan ^{-1}\left\{\frac{\sqrt{B_{y}^{2}+B_{z}^{2}}}{B_{x}}\right\}$,

is shown in Fig. 2E. The first thing to notice is that over the whole tail excursion the cone angle varies over $0^{\circ} \leq \theta_{\mathrm{c}} \leq 180^{\circ}$, where $0^{\circ}$ is sunward field and $180^{\circ}$ is anti-sunward. There is no clear pattern in the cone angle. In Fig. 3 the cone angle is colour coded along the orbit of Rosetta, projected on the $X \rho$ plane, where $\rho=\sqrt{Y^{2}+Z^{2}}$ in the middle panel, and projected onto the $Y Z$-plane in the bottom panel.

Theoretically, the change from sunward to anti-sunward directed magnetic field should occur at the line $Y=0$, or slightly abberated for solar wind direction, because this line is the centre of the draped magnetotail. However, it is clear in the bottom panel that this is not the case, where a change of colour would be expected from mainly blue on one side to mainly red at the other.

In order to study whether ideal draping directions occur, two $30^{\circ}$-intervals of $\theta_{\mathrm{c}}$ are investigated. For the whole interval discussed here, there are $\sim 10000$ points with $\theta_{\mathrm{c}} \leq 30^{\circ}$ and only $\sim 380$ points with $\theta_{\mathrm{c}} \geq 150^{\circ}$. In contrast, for cone angles $75^{\circ} \leq \theta_{\mathrm{c}} \leq 105^{\circ}$ a total of $\sim 23000$ points were found. Clearly, the near-tail has a completely different structure than simple draped fields.

Another interesting thing is found when looking at the location of the different field directions. The anti-sunward directed field only occurs at the beginning of the interval on 19 March 2016 within $R \sim 15 \mathrm{~km}$. The sunward directed field is more spread out over the interval and is mainly occurring for radial distances $\rho \geq 100 \mathrm{~km}$ from the comet-sun line.

As the propagation model by Tao et al. (2005) only delivers the tangential magnetic field at comet 67P, the OMNI data are used to calculate the cone angle, OMNI $\theta_{c}$, of the solar wind, and the propagation time from the ballistic model (Opitz et al. 2009, 2010), on average six days, is used. The result is shown in Fig. $2 \mathrm{~K}$. Although there are variations on OMNI $\theta_{\mathrm{c}}$, the larger picture shows gradual changes from anti-sunward to sunward, etc. This behaviour is not reflected in the RPC $\theta_{\mathrm{c}}$, Fig. $2 \mathrm{E}$, which means that the weakly outgassing comet has an influence on the magnetic field structure.

To further study the behaviour of the cone angle, a histogram of the occurrence percentage of angles is shown in Fig. 4 for all MAG data (blue) and for high-field data (red), both normalized to the total number of data points, i.e. red does not sum up to $100 \%$. At $2.7 \mathrm{AU}$ distance from the Sun of comet 67P, the expected Parker spiral angle is $\theta_{\mathrm{P}} \approx 60^{\circ}$, which is added as a vertical black line at both 60 and 120 for sunward and anti-sunward pointing magnetic field, respectively. Both sets of angles peak at angles of $\theta_{\mathrm{c}} \approx 70^{\circ}$, whereas there is a minor peak in the opposite direction $\theta_{\mathrm{c}} \approx 110^{\circ}$.

In contrast, in the bottom panel of Fig. 4 the histogram for the OMNI data (6 days time shifted) are represented, which show that at Earth there is regular distribution of the cone angle, with equal peaks near $45^{\circ}$ and $135^{\circ}$, expected for a period of about one synodic solar rotation ( $\sim 27$ days for Rosetta). It should also be noted that the heliographic latitude difference of $5.1^{\circ}$ between the Earth and Rosetta may play a small role in difference of the cone-angle distribution.

This means that, although the comet is not very active, it still is having an effect on the solar wind. Somehow sunward
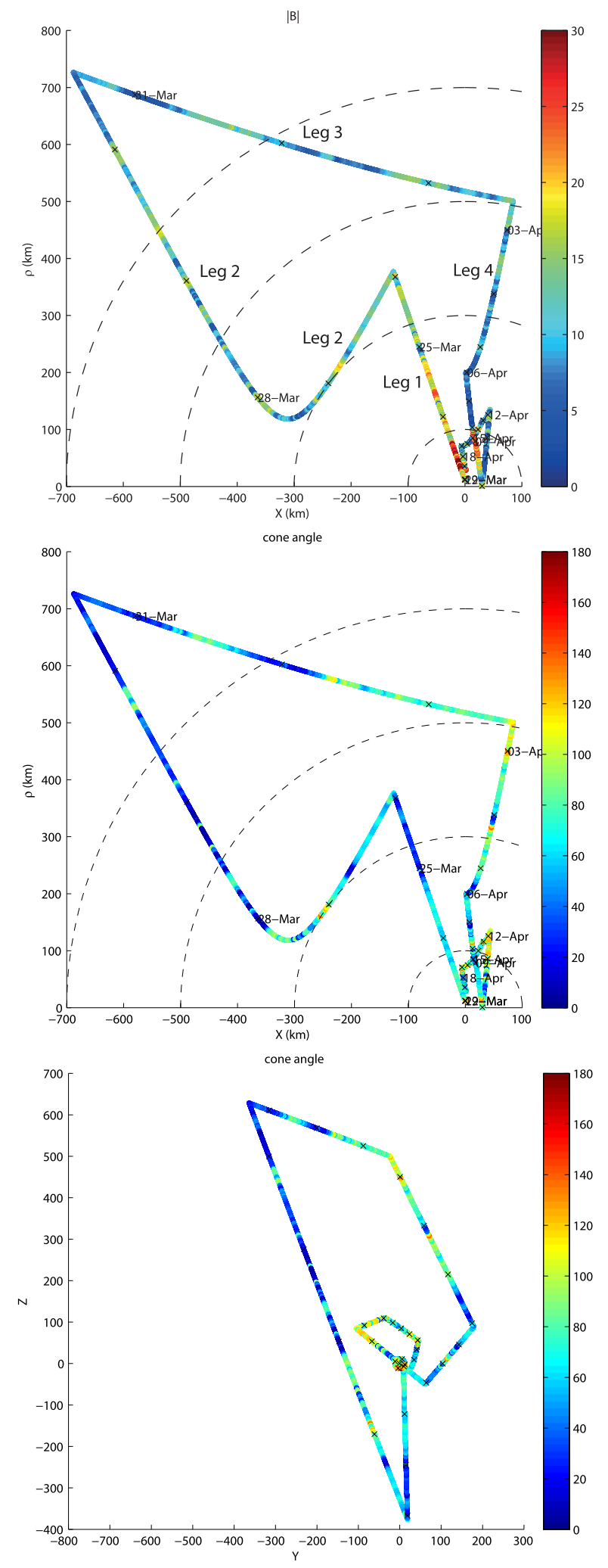

Fig. 3. Top two panels: magnetic field strength $|B|$ and cone angle $\theta_{\mathrm{c}}$ along the orbit of the tail excursion in cylindrical coordinates $X_{\mathrm{CSEQ}}$ and $\rho=\sqrt{Y_{\mathrm{CSEQ}}^{2}+Z_{\mathrm{CSEQ}}^{2}}$. The dashed lines are circles of constant radial distance $R$ from the comet. The orbit is split up into four legs: Leg 1 is shown radially away from the comet; Leg 2 is a curved path to apocentre; and Leg 3 and Leg 4 bring the spacecraft back to the comet (for the specific intervals see Table 1). Bottom panel: cone angle along the orbit projected onto the $Y Z$-plane. 
Table 1. Four intervals for which the behaviour of the magnetic field strength is investigated.

\begin{tabular}{llccccccc}
\hline \hline & & \multicolumn{3}{c}{ Power law } & \multicolumn{3}{c}{ Polynomial } \\
Leg & Interval & $B_{0}$ & $\alpha$ & $\mathcal{R}^{2}$ & Range & $B_{1}$ & $\beta$ & $\mathcal{R}^{2}$ \\
\hline Leg 1 & 22-26 March & $37 \pm 1$ & $-0.15 \pm 0.005$ & 0.36 & $10-400$ & NA & NA & NA \\
Leg 2 & 26-30.5 March & $25 \pm 2$ & $-0.13 \pm 0.02$ & 0.02 & $300-1000$ & NA & NA & NA \\
Leg 3 & 30.5 March-3 April & NA & NA & NA & $450-1000$ & $14 \pm 0.3$ & $-0.0062 \pm 0.0004$ & 0.01 \\
Leg 4 & 3 April-7 April & NA & NA & NA & $150-450$ & $2.5 \pm 0.1$ & $0.018 \pm 0.005$ & 0.30 \\
\hline
\end{tabular}

Notes. Investigated fits are $B=B_{0} R^{\alpha}$ and $B=B_{1}+\beta R$. For the fits the goodness-of-fit is given by $\mathcal{R}^{2}$.

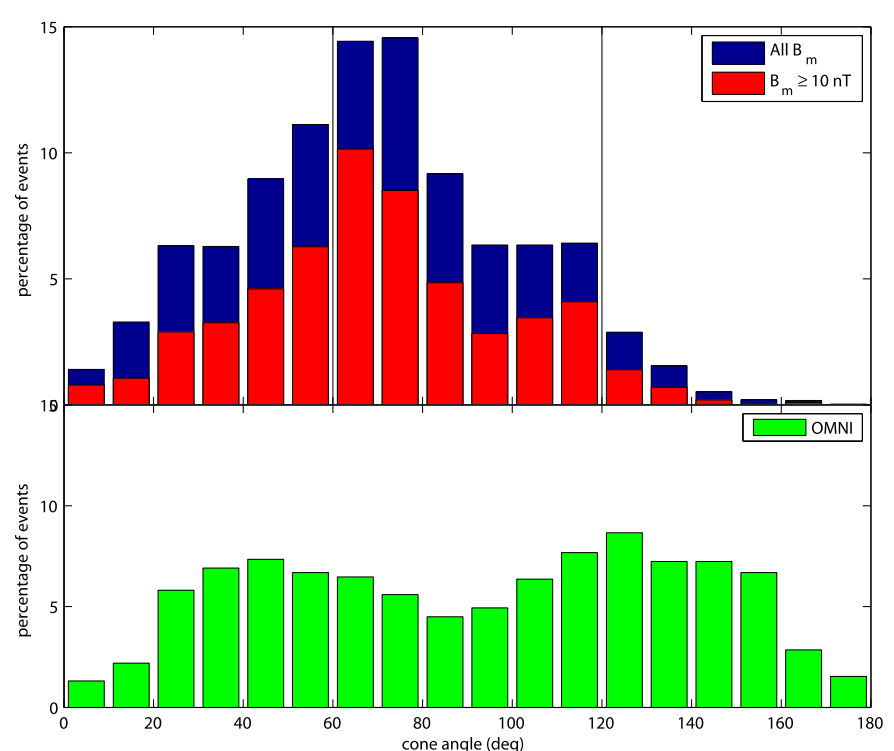

Fig. 4. Histogram of the occurrence percentage of the cone angle $\theta_{\mathrm{c}}$. Top panel: values for all RPC MAG data (blue) and for high-field $B_{\mathrm{m}} \geq$ $10 \mathrm{nT}$ data (red). The two vertical lines show the estimated Parker spiral values $\theta \approx 60^{\circ}$ for Rosetta at $\sim 2.7$ AU. Bottom panel: values for the OMNI data.

and transverse magnetic fields are favoured in the near tail region, whereas at Earth the solar wind has a regular distribution of angles. We note that there is an assumption that the data are correctly calibrated, which might not be the case. The consequences of remaining errors in the offset of the magnetometer are investigated in the discussion section.

\subsection{Fitting $B_{\mathrm{m}}$}

At the upstream side of the comet the magnetic field strength was theoretically expected to decrease exponentially with distance from the nucleus (see e.g. Koenders et al. 2015, Fig. 3). However, Goetz et al. (2017) showed that in the inner region of the coma of comet $67 \mathrm{P}$, i.e. $10 \leq R \leq 1000 \mathrm{~km}$, the magnetic field mainly remains constant, which could be explained by regular magnetic pile-up of the shocked and mass-loaded solar wind magnetic field, to which a plasma cooling through chargeexchange term is added as discussed by Galeev et al. (1985).

For the downstream side, the situation can be different. As the tail excursion consists of four separate legs, the data are divided into four sub-intervals as listed in Table 1. Per leg the data is fitted either by a power law or by a first-order polynomial, depending on the behaviour of the field magnitude $B_{\mathrm{m}}$. The results of the fitting are shown in Table 1 and Fig. 5.
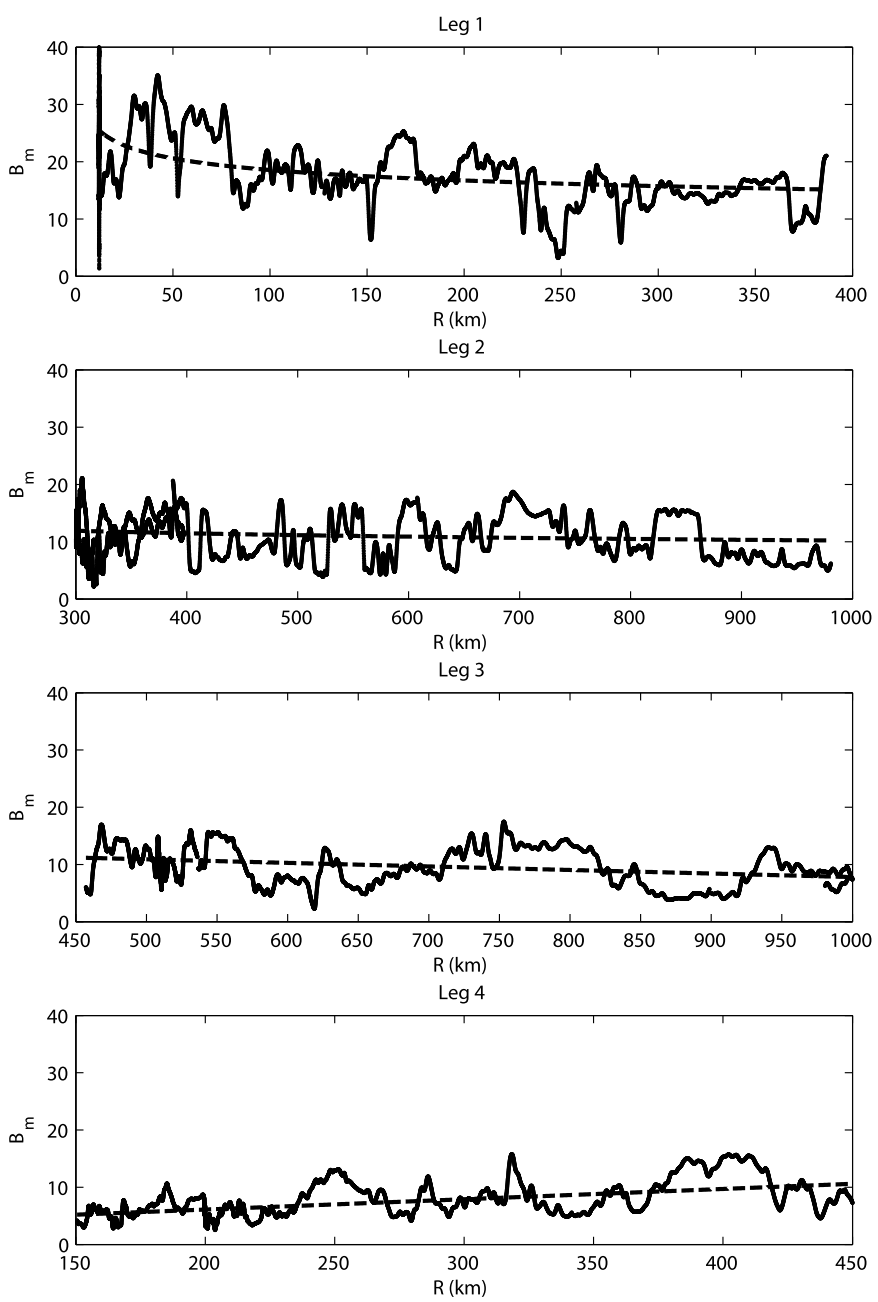

Fig. 5. Magnetic field strength, $B_{\mathrm{m}}$, during the four legs of the tail excursion. The dashed lines show the fits of $B_{\mathrm{m}}$ as functions of radial distance from the comet, $R$, with the fit parameters shown in Table 1 .

It is clear from the fits that there is no simple behaviour of the magnetic field strength along the legs of the tail excursion, but the $\mathcal{R}^{2}$ values for goodness-of-fit are very bad, which means that only a trend can be indicated by these fits. The first two legs are fitted with a power law and show a comparable fall-off with distance $\alpha=-0.15 /-0.13$ albeit with different values for $B_{0}$. However, the magnetic field strength $B_{\mathrm{m}}$ behaves rather differently for the third and fourth leg. During Leg 3 the field is better described by a linear fit that has decreasing $B_{\mathrm{m}}$ with increasing distance. The fourth leg shows an unexpected behaviour, where the linear fit shows a decreasing $B_{\mathrm{m}}$ with decreasing distance from the comet, although it can easily be argued that the field 


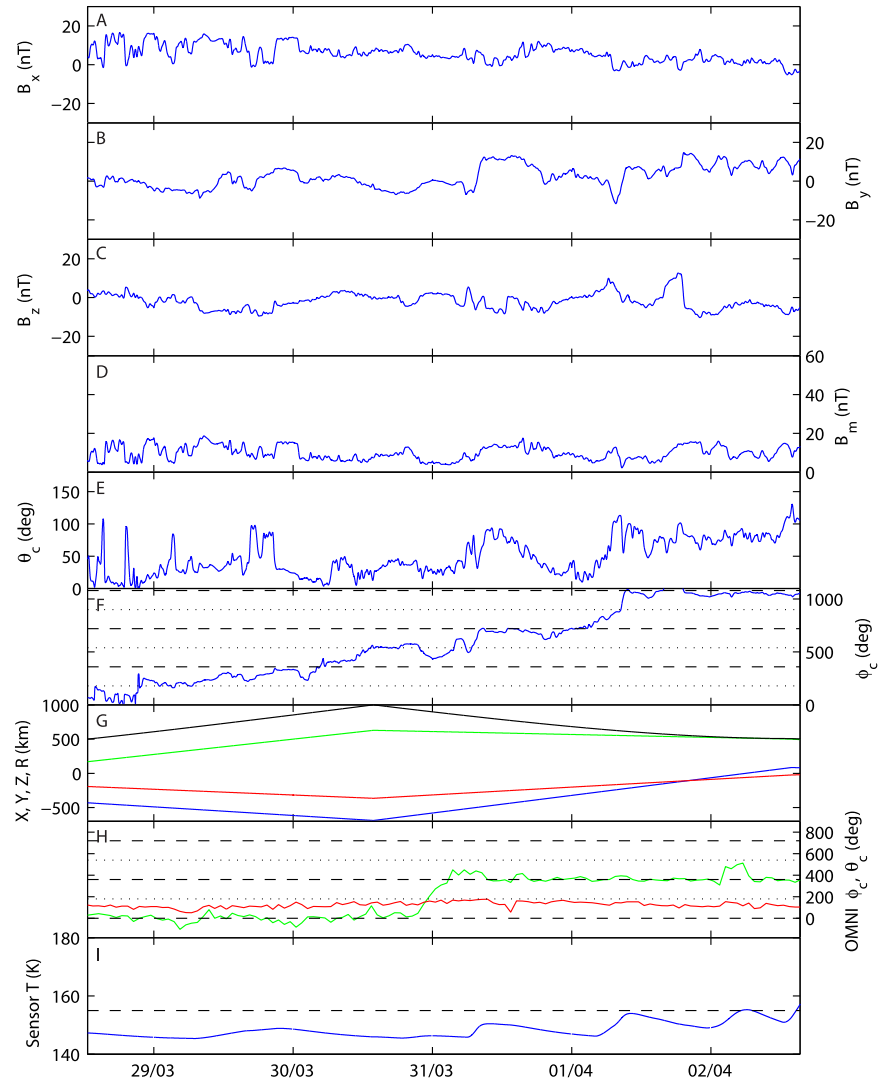

Fig. 6. Far-field part of the tail excursion $(R \geq 500 \mathrm{~km})$. (A-D) The components and total magnetic field; $(E)$ the con-angle $\theta_{c} ;(F)$ the unwrapped clock angle $\phi_{c} ;(G)$ the location of Rosetta $X$ (blue), $Y$ (red), $Z$ (green), and $R$ (black); $(H)$ the unwrapped OMNI clock angle (green) and the cone angle (red); and $(I)$ the sensor temperature.

strength remains constant. As numerical simulations for the tail excursion become available, this behaviour of the magnetic field should be checked.

\subsection{Clock angle behaviour}

In order to further study the structure of the tail, a more-orless undisturbed part of the tail excursion is chosen, i.e. for $R>500 \mathrm{~km}$ (see Fig. 8 to be discussed in the next section). The averaged magnetic field as shown in Fig. 2 is zoomed in Fig. 6, in which case the clock angle of the magnetic field in the tail, defined as

$\phi_{\mathrm{c}}=\tan ^{-1}\left\{\frac{B_{z}}{B_{y}}\right\}$

is unwrapped, i.e. absolute jumps greater than or equal to $\pi$ are changed to their $2 \pi$ complement.

The first thing to notice in the magnetic field structure for $R>500 \mathrm{~km}$ is that the clock angle $\phi_{\mathrm{c}}$ is overall gradually increasing from $\phi_{\mathrm{c}}=0^{\circ}$ to $\phi_{\mathrm{c}}=1080^{\circ}$, with some decreases on 30 and 31 March, and a long plateau covering the last part of 31 March, which seems to coincide with little motion and even a small reduction in the radial distance and remains flat after rotating once more over $360^{\circ}$ during 1 April.

Although the distance of Rosetta to comet 67P increases and decreases during this part of the tail excursion, Fig. 6G, the clock angle continues to increase. However, there is a difference in behaviour for the pre- and post-apogee parts of the tail excursion.

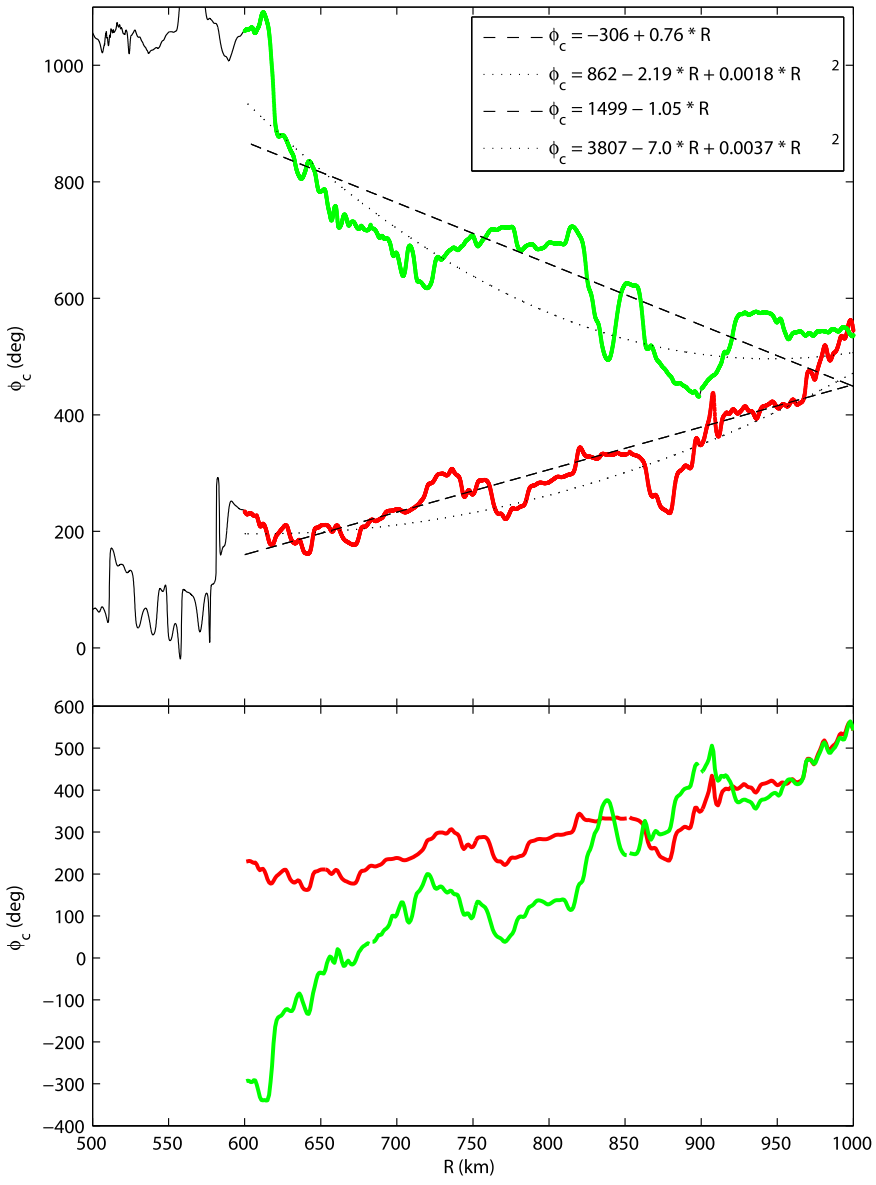

Fig. 7. Top: clock angle, $\phi_{\mathrm{c}}$ for the far field $(R \geq 500 \mathrm{~km})$ plotted as a function of radial distance $R$. For pre- (red) and post-apogee (green) the variation of $\phi_{\mathrm{c}}$ is fitted up to $R=600 \mathrm{~km}$ with a linear (dashed) and quadratic (dotted) fit. The fitting results are listed in the legend. Bottom: clock angle $\phi_{\mathrm{c}}$ for the outbound leg as in top panel, where the clock angle is rewinding for the inbound leg.

Both the pre- and post-apogee clock angles are fitted for $R \geq 600 \mathrm{~km}$ with a $1 \mathrm{st}$ and 2 nd order polynomial. The result of the fits are shown in Fig. 7 top panel. It is clear that for the post-apogee leg (green) the increase in clock angle is much faster than for pre-apogee (red). The different rate in change of clock angle is, however, not reflected in the time domain. The preapogee leg (red) lasts for $\sim 49 \mathrm{~h}$ and the 2 nd order fit gives a total rotation of $\Delta \phi_{\mathrm{c}} \approx 277^{\circ}$, which leads to a angular frequency of $\omega_{\mathrm{h}} \sim 5.65^{\circ} \mathrm{h}^{-1}$. The post-apogee leg (green) lasts for $\sim 74 \mathrm{~h}$ and the 2nd order fit give a total rotation of $\Delta \phi_{\mathrm{c}} \approx 432^{\circ}$, which results in an angular frequency of $\omega_{\mathrm{h}} \sim 5.83^{\circ} \mathrm{h}^{-1}$. These differences in angular frequency and the different spatial rate of clock-angle change could imply that there is a rotational structure moving along the tail winding the magnetic field, which first overtakes Rosetta on the pre-apogee leg. After apogee, with Rosetta moving towards comet 67P, the spacecraft is running into this structure, thereby observing an accelerated increase in clock-angle $\phi_{\mathrm{c}}$. This is further discussed in the discussion section below.

In Fig. 7 bottom panel the clock-angle $\phi_{\mathrm{c}}$ for the outbound leg (red) is plotted as in the top panel. For the inbound leg (green) the clock angle is rewound showing that for both parts, outside of $R \approx 700 \mathrm{~km}$, the same structures are found, slightly modified, which can be a result of the different paths through the tail. Within $R \approx 700 \mathrm{~km}$ the clock angle rotates much faster for 
the inbound leg. A clearly different structure is found between $800 \leq R \leq 850 \mathrm{~km}$, where the outbound (red) curve is flat, whereas the inbound (green) curve shows a peaked structure.

Similar as in the cone-angle case, the OMNI clock angle shows a very different behaviour when compared with the MAG data. In Fig. $6 \mathrm{H}$ the unwrapped OMNI $\phi_{\mathrm{c}}$ is plotted, which shows a constant $\phi_{\mathrm{c}} \approx 0^{\circ}$ until late 30 March, after which $\phi_{\mathrm{c}}$ rotates over $\sim 360^{\circ}$ and then remains constant at $\phi_{\mathrm{c}} \approx 360^{\circ}$. Although it might be difficult to discern in Fig. $6 \mathrm{H}$, the rotation of the clock angle happens near the maximum in the cone angle.

\section{Near tail - small scale dynamics}

One of the most characteristic small scale phenomena in the RPC MAG data was the singing comet (Richter et al. 2015, 2016), quasi-monochromatic waves within the frequency range of $10 \leq f_{\text {sing }} \leq 100 \mathrm{mHz}$. Other small scale structures were also observed, for example current sheets between oppositely directed draped magnetic fields in the coma (Volwerk et al. 2017), which are not discussed in this paper.

\subsection{The singing comet}

The singing comet, prominent in the early phase of Rosetta's mission, was observed until spring 2015, when comet $67 \mathrm{P}$ was more than $\sim 2.2 \mathrm{AU}$ from the Sun. After that it is unclear whether the instability disappeared or that the signature was swamped in the strongly active magnetic environment around perihelion (see also Glassmeier 2017). However, approximately six months after perihelion (13 August 2015) the singing comet reappeared again in the RPC MAG data.

In order to study the singing comet signature, the data are transformed to a mean-field aligned coordinate system, in which low-pass filtered data for periods longer than $1 \mathrm{~h}$ are used as the new $\mathbf{Z}_{\mathrm{MFA}}$ coordinate. The new $Y$-direction is defined as $Y_{\mathrm{MFA}}=$ $\mathbf{R} \times \mathbf{Z}_{\text {MFA }}$, where $\mathbf{R}$ is the position of Rosetta in CSEQ coordinates, and finally $X_{\mathrm{MFA}}$ completes the right-handed system and is mainly along the radial direction $\mathbf{R}$. The two transverse components of the MFA magnetic field are then transformed into rightand a left-hand polarized components: $L / R=0.5\left(X_{\mathrm{MFA}} \pm i Y_{\mathrm{MFA}}\right)$. Spectral analysis is performed on the three components of the MFA data $(R, L$, and $C)$ in sliding windows of $512 \mathrm{~s}$ with a shift of $30 \mathrm{~s}$. For each power spectrum the power is summed over the interval $10 \leq f_{\text {sing }} \leq 100 \mathrm{mHz}$ and the frequency of peak energy is determined as $f_{\max }$ for which $P\left(f_{\max }\right)=P_{\max }$. The result is shown in Fig. 8. Also, for each of these windows a minimum variance analysis (MVA; Sonnerup \& Scheible 1998) is performed on the magnetic field.

Overall, the median peak frequency along the tail excursion is $f_{\text {sing,med }} \approx 15 \mathrm{mHz}$ and the mean $f_{\text {sing,mean }} \approx 19 \mathrm{mHz}$, which is lower than the original peak around $40 \mathrm{mHz}$. The maximum peak frequency is $f_{\text {sing, } \max } \approx 99 \mathrm{mHz}$.

The constant radial distance circles in Fig. 8 show that the main power in the waves is located within $700 \mathrm{~km}$ radial distance. However, this is only true for the outbound leg; there is very little power in the inbound leg, after the turning point at $(X, \rho) \approx(-700,700) \mathrm{km}$, even after the $R=700 \mathrm{~km}$ circle is crossed. Indeed, during the inbound leg Rosetta remains near $Z \approx 600 \mathrm{~km}$ for a long time, which seems to be a region in which the singing comet is very inactive. This probably means that the instability generating the singing-comet waves (Meier et al. 2016) is less productive, which could be caused by a reduction in freshly pick-up ions and/or lower velocities of the pick-up ions for the outer part of Leg 2 and for Legs 3 and 4.
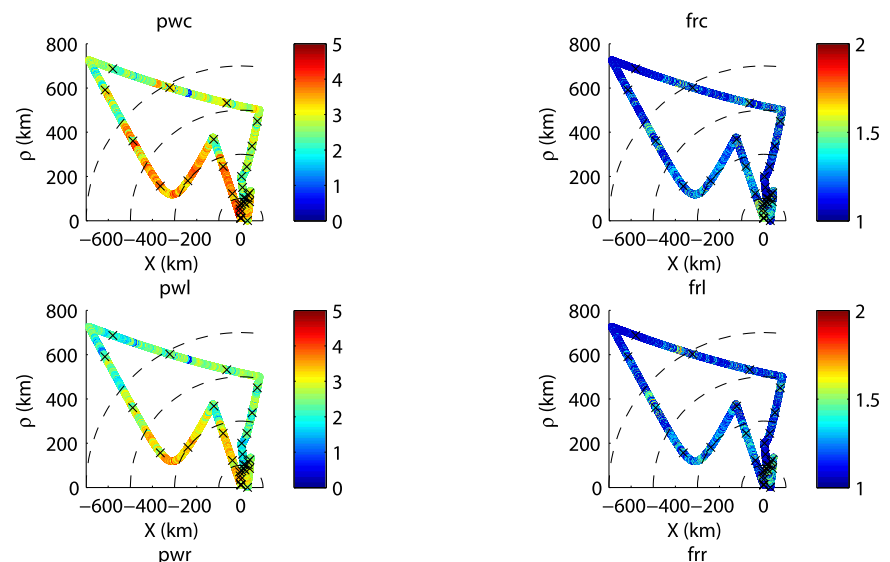

$\mathrm{X}(\mathrm{km})$
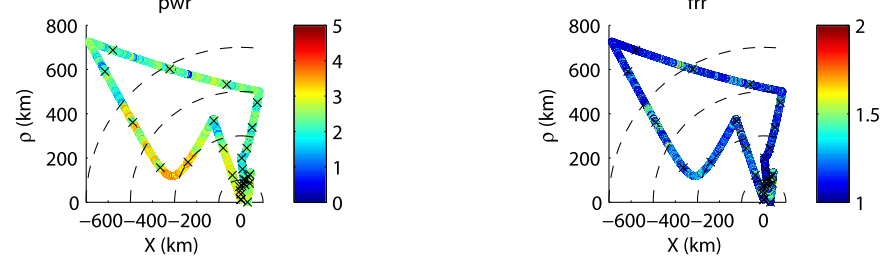

Fig. 8. Left column: power in the singing-comet frequency band for the compressional and left- and right-hand polarized components of the magnetic field. Right column: frequency at maximum power.

Another possibility is that this lull in singing power is caused by kinetic plasma effects. Assuming that the magnetic field is mainly in one direction, which is validated by the histograms, then, according to numerical simulations for weakly outgassing comet 67P (Koenders et al. 2015, 2016), the influence of the presence of the comet should only be seen in one hemisphere. This would fit with the orbit of Rosetta.

Using minimum variance analysis (Sonnerup \& Scheible 1998) the propagation direction of the singing comet waves is determined for each sliding window in the CSEQ coordinate system, including the ratio of the intermediate and minimum eigen value. The angle $\alpha_{\text {mvb }}$ between minimum variance direction and mean magnetic field within the window is calculated along with the angle $\beta_{\mathrm{mvr}}$ between minimum variance direction and radial direction to the comet. The results are shown in Fig. 9.

Although all angles $\alpha_{\mathrm{mvb}}$ and $\beta_{\mathrm{mvr}}$ are plotted in blue for the intervals Leg 1 and 2, not all values should be taken as reliable. Therefore, the angles for a ratio $\lambda_{2} / \lambda_{1}>3$ are plotted in red, which show that $\alpha_{\mathrm{mvb}} \approx 79^{\circ} \pm 24^{\circ}$ and $\beta_{\mathrm{mvr}} \approx 55^{\circ} \pm 19^{\circ}$. The value found for $\alpha_{\mathrm{mvb}}$ agrees well with the values found by Richter et al. (2016) during the Philae landing.

In Fig. 9D the minimum variance direction is plotted, which also shows an interesting pattern. All MVA estimates are shown in grey, but for eigen value ratios $\lambda_{2} / \lambda_{1}>3$ the $X, Y$, and $Z$ component of the minimum eigen vector are indicated in blue, red, and green dots, respectively. Three different, relatively stable regions can be discerned for which the propagation direction of the singing waves are

$$
\begin{aligned}
& \text { - 22-23 March: }(0.80,0.31,0.40) \pm(0.11,0.19,0.24) \text {; } \\
& \text { - 24-27 March: }(0.56,0.10,-0.73) \pm(0.18,0.30,0.18) \text {; } \\
& \text { - 28-30 March: }(0.39,-0.32,0.76) \pm(0.18,0.31,0.20) \text {. }
\end{aligned}
$$

\subsection{Turbulent cascade of energy}

The power in the singing comet waves has to be dissipated, which usually in a plasma occurs through turbulent cascade (see e.g. Vörös et al. 2004; Volwerk et al. 2004). Therefore, during the 


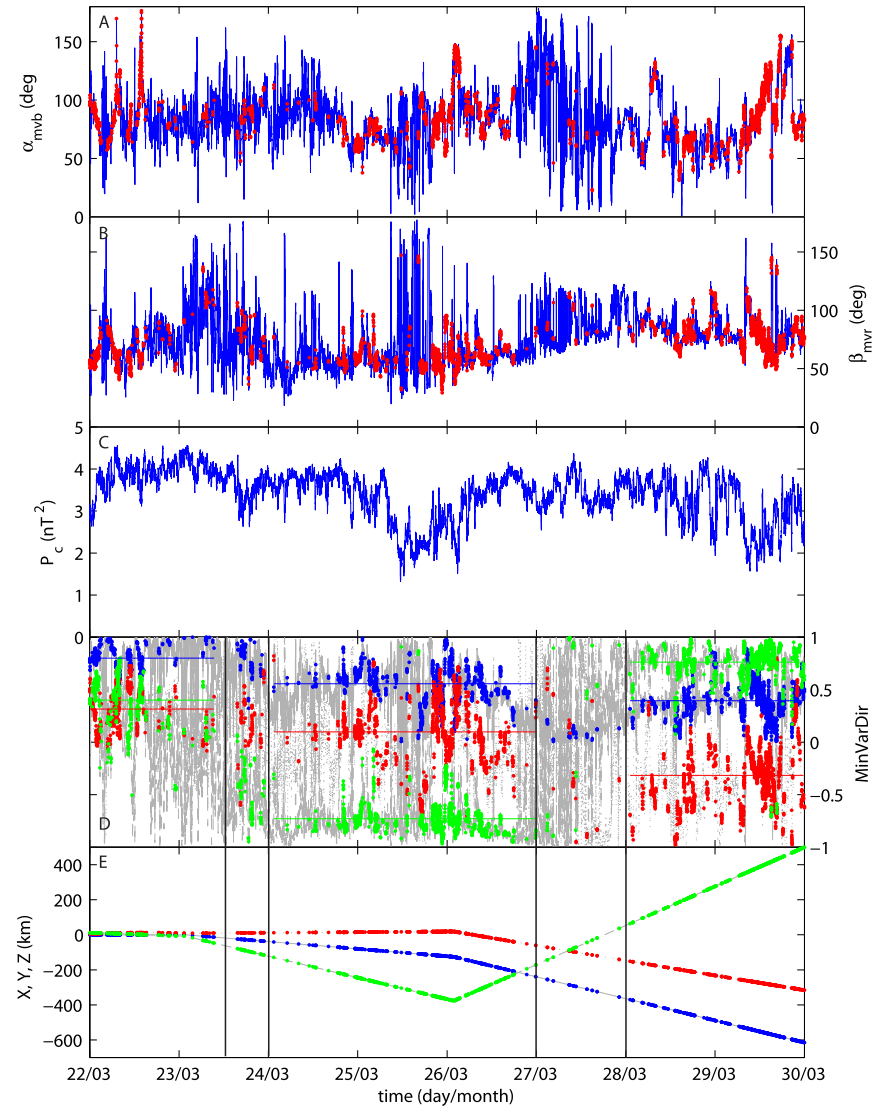

Fig. 9. Zoom-in on the most active singing part of the tail excursion. (A) The angle $\alpha_{\text {mvb }}$ between the minimum variance direction and magnetic field direction for the whole interval in blue and the red dots show where the minimum variance analysis can be trusted with $\lambda_{2} / \lambda_{1}>3 ;(B)$ the angle $\beta_{\mathrm{mvr}}$ between the minimum variance direction and the radial direction to the comet; $(C)$ the total power in the singing comet frequency band; $(D)$ the minimum variance direction $X$ (blue), $Y$ (red) and $Z$ (green) components for $\lambda_{2} / \lambda_{1}>3$ with the mean value for three intervals overplotted; and $(E)$ the location of Rosetta $X$ (blue), $Y$ (red) and $Z$ (green). The black vertical lines indicate the relatively stable MVA intervals mentioned in the text.

spectral analysis, presented in the previous section, the trace of the power spectral matrix was fitted to a power law in frequency

$P_{f_{1}}^{f_{\mathrm{h}}}(f)=P_{0} f^{\alpha}$,

within boundaries given by the frequency of maximum power spectral density $f_{\text {sing,max }}$ as low frequency $f_{1}$ and $250 \mathrm{mHz}$ as the high frequency $f_{\mathrm{h}}$. From visual inspection the spectral power density flattens for frequencies higher than $250 \mathrm{mHz}$. Naturally, not all spectra can be fitted well, and therefore, only fits with a value of $\mathcal{R}^{2} \geq 0.95$ are investigated. The result is shown in Fig. 10, where the slope $\alpha$ is plotted as a function of distance from the comet for the singing dominated interval at the beginning of the tail excursion (until 29 March 2016) and the no singing interval that makes up the rest of the tail excursion.

It is clear that there is a large spread in the spectral index $\alpha$ in Fig. 10. For bins of $100 \mathrm{~km}$ in radial distance $R$ from the comet, the median (red), mean (green), and standard deviation (blue) are plotted. A slight trend can be seen in the data: for the singing the average spectral index $-2.2 \leq \bar{\alpha} \leq-2$, whereas for the nonsinging the average spectral index $-1.8 \leq \bar{\alpha} \leq-1.5$ (omitting the first two boxes $0-200 \mathrm{~km}$ ).
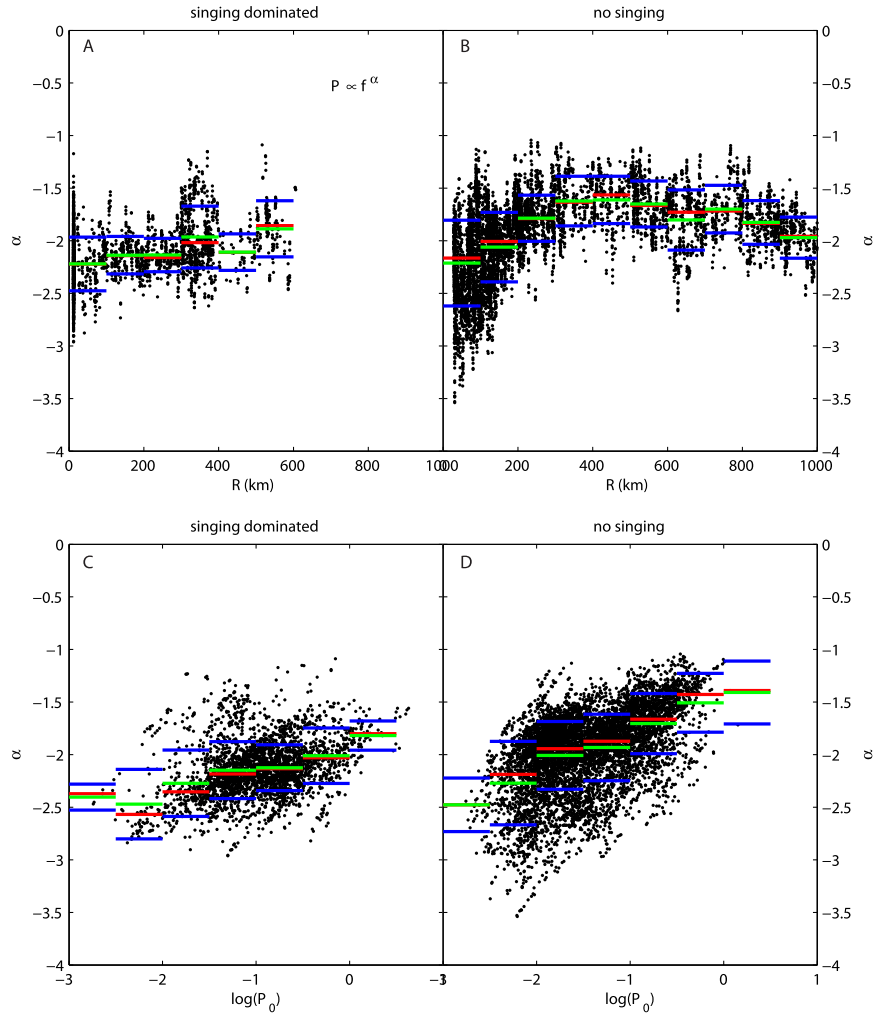

Fig. 10. Panels $A$ and $B$ : spectral index $\alpha$ from the power law fittings (with $\mathcal{R}^{2}>0.95$ ) as a function of radial distance from the comet for the singing dominated intervals and the no singing intervals. For a bin size of $100 \mathrm{~km}$ the median (red), mean (green), and standard deviation (blue) of the $\alpha$-distribution are plotted. Panels $C$ and $D$ : spectral index $\alpha$ as a function of $\log \left(P_{0}\right)$ from the power law fittings. For a bin size of 1 the median (red), mean (green), and standard deviation (blue) of the $\alpha$-distribution are plotted.

For the pristine solar wind and long time intervals the spectral index is usually found to be that of Kolmogorov turbulence with $\alpha=-5 / 3$ (Kolmogorov 1941). However, for short time intervals and in the presence of magnetoplasma structures the spectral index can easily vary between $-2 \leq \alpha \leq-1$ (see e.g. Wicks et al. 2010; Schekochihin 2017).

For the same set of well-defined fits, the relationship between $\alpha$ and $P_{0}$ is also investigated and shown in the bottom panels of Fig. 10. Although there is a large spread in the values of $\alpha$ there is clear trend that the spectrum gets flatter with increasing $P_{0}$. When there is strong energy input at frequency $f_{1}$ then there is a strong cascade to higher frequencies, spreading the energy, and thereby flattening the spectrum.

\section{Discussion}

The RPC MAG observations during the Rosetta tail excursion have been studied in this paper, first looking at the large scale structure of the tail and then at small scale processes as the singing comet and the turbulent cascade of the energy input into the magnetic field oscillations. Owing to the lateness in the mission of this excursion, the comet was already a lot less active, which influences its interaction with the solar wind; for example, the magnetic field line draping is much less pronounced for these conditions. The orbit of the tail excursion was chosen in such a way that different regions of comet 67P's tail could be sampled, i.e. crossing of both the ion and dust tail regions. 
Field line draping around an active comet was first described by Alfvén (1957), where the interaction of a strongly outgassing comet with the solar wind magnetoplasma creates a well-established cometary magnetotail, as was observed by ICE at comet 27P/Giacobini-Zinner (Slavin et al. 1986). However, this clear interaction does not take place at an weakly outgassing comet such as 67P. Instead of the expected field line draping in the $X Y$-plane it was found that on 28 March 2015 the field line draping was mainly in the $Y Z$-plane (see Fig. 2 in Koenders et al. 2016). This remarkable feature was explained using numerical simulations, which showed that because of the strong deflection of the plasma flow near the comet, where the freshly pickedup ions move in the direction of the convective electric field of the solar wind, the solar wind ions and its magnetic field move in the opposite direction to maintain momentum balance (Newton's 2nd law), the draping mainly occurs in the $Y Z$-plane (see Fig. 5 in Koenders et al. 2016).

During the tail excursion, the activity of the comet is much lower again as around perihelion by about a factor $10\left(Q \sim 10^{25}\right.$ vs. $10^{26} \mathrm{~s}^{-1}$, Hansen et al. 2016), which means less mass loading of the solar wind and thus a lesser field line draping as an effect. The MAG observations presented in this paper show that the cone angle during the tail excursion are mainly $\theta_{\mathrm{c}} \leq 90^{\circ}$, strongly different from the IMF from the OMNI data, timeshifted to represent the same interval. So, what causes the completely different distributions of $\theta_{\mathrm{c}}$ shown in Fig. 4? This could either be a real physical effect caused by the comet or it may be a artificial effect due to inadequate offset determination caused by the low sensor temperatures.

Up to now it has been assumed that the data are fully calibrated, although it was commented above that the sensor temperature was often outside the validity range of the thermal model used by Goetz et al. (2017). It has been shown by Plaschke et al. (2017) that a residual offset could be determined for the MAG data after calibration as described by Goetz et al. (2016a) at a value of $\mathbf{O}_{\text {diff }}=(4.16,-0.47,-3.06) n T$. In order to check what effect this offset has on the cone-angle determination in the tail, and whether it can explain the different distribution as the solar wind cone angle, half the offset is added/subtracted from the MAG data used in this paper and the cone angle is redetermined. The result is shown in the top histogram in Fig. 11. Adding the offset to the MAG data, however, makes the correspondence between the MAG and OMNI histograms even worse: subtracting $\mathbf{O}_{\text {diff }}$ from the MAG data slightly improves the correspondence (however, this would be correcting the data in the wrong direction), as can be seen by comparing Figs. 4 bottom panel and 11 top panel.

Another error estimate in the cone angle can be obtained by assuming that the sensors have an error of $\pm 5 \mathrm{nT}$, then the error in the cone angle can be determined by

$\Delta \theta_{\mathrm{c}}=\Sigma_{i} \frac{\partial}{\partial x_{i}} \tan ^{-1}\left\{\frac{\sqrt{B_{y}^{2}+B_{z}^{2}}}{B_{x}}\right\} \Delta B_{i}$.

Again, a histogram of the clock angle is prepared for the three cases: $\theta_{c}-\Delta \theta, \theta_{c}$, and $\theta_{c}+\Delta \theta$, which is shown in Fig. 11 bottom panel. As in the offset case, the histograms do not converge to the clock-angle distribution of the IMF.

In all, taking into account possible remaining errors in the offset of RPC-MAG and the result in the cone-angle $\theta_{c}$ distribution shows that there is neither the incoming solar wind cone-angle bi-peaked distribution near the Parker spiral angle,

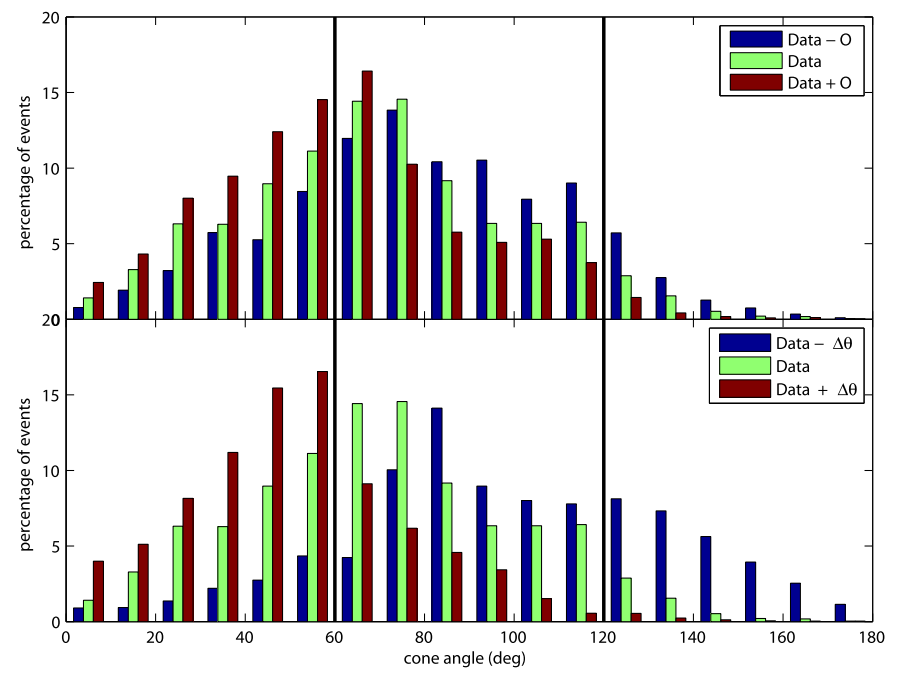

Fig. 11. Histogram of the occurrence percentage of the cone angle, $\theta_{\mathrm{c}}$ as in Fig. 4 with $\mathbf{O}_{\text {diff }}$ added to the MAG data (top) and subtracted from the MAG data (bottom).

nor is there the bi-directional distribution of a fully draped magnetotail expected to peak around small $\left(\leq 30^{\circ}\right)$ and large $\left(\geq 150^{\circ}\right)$ cone angles. This indicates an influence of the weakly outgassing comet 67P on the IMF, which is decidedly different from previously encountered strongly outgassing comets.

If the cone angle effect is not an offset issue, then comet 67P should influence the solar wind magnetic field even though it is only outgassing weakly. That the magnetic field is not well ordered should not come as a big surprise. It was shown that the nested draping in the coma of comet $67 \mathrm{P}$ is highly variable, as discussed by Volwerk et al. (2017), with the direction of the field changing on the timescale of about $1 \mathrm{~h}$. The coma is much less organized than the fields measured at comet 1P/Halley (see e.g. Riedler et al. 1986; Raeder et al. 1987), but this may well be an effect from the enormous differences in fly-by velocities of the spacecraft and the huge amount of data available from the ROSETTA mission over a time period of more than two years.

One way of obtaining an unbalanced cone-angle distribution from a balanced solar wind would be if the comet were surrounded by an asymmetric gas cloud, in which more pick-up occurred on the leading side of comet 67P. Dominating pick-up at $Y<0$ would favour sunward bending of the IMF field lines, thereby constructing an asymmetry in the draped field in the tail. The numerical simulations for a more active comet 67P at $2 \mathrm{AU}$ by Koenders et al. (2016, Fig. 3e and f) indicate a strong asymmetry in the solar wind ion density $n_{\mathrm{SW}}$ and a weak asymmetry in the cometary ion density $n_{\mathrm{CI}}$ in the $Y$ direction.

Not only did the cone angle show peculiar behaviour, the clock angle, at radial distances $R>600 \mathrm{~km}$, also showed strong differences from the OMNI clock angle. The value $\phi_{\mathrm{c}}$ for RPC MAG increased continuously over $\sim 900^{\circ}$, whereas OMNI $\phi_{\mathrm{c}}$ only showed a $\sim 360^{\circ}$ rotation over a short interval and remained constant the rest of the time. Again including the offset as for the cone-angle case did not produce any significant changes, apart from raising or lowering the absolute values of $\phi_{c}$. Interestingly, applying the offset leads to the opposite result as in the clock-angle case, in which adding $\mathbf{O}_{\text {diff }}$ brings RPC MAG $\phi_{\mathrm{c}}$ closer to OMNI $\phi_{\mathrm{c}}$, whereas subtracting it moves it further away.

The continuously increasing clock-angle $\phi_{\mathrm{c}}$ means that the magnetic field of the tail is wound-up, where for the outbound 
pass of Rosetta (red in Fig. 7) the radial gradient of the clock angle is smaller than for the inbound pass (green). This behaviour can be explained for a winding wave passing along the tail, with a phase speed faster than Rossetta's velocity, which overtakes the spacecraft. This kind of structure is reminiscent of the helical waves in cometary tails that were first observed at comet Kohoutek (C/1973 E1, 1973 XII, or 1973f) by Hyder et al. (1974). It was assumed that this was created by a kink-like instability created by the current flowing along the field lines down the tail (see also Ip \& Mendis 1975). Later Ershkovich \& Heller (1977) also discussed these waves in tails of comets Morehouse (C/1908 R1) and Arend-Roland (C/1965 R1). By modelling the cometary tail as a cylinder they found the dispersion equation for helical oscillations and that the phase velocity was close to the estimated Alfvén velocity. It should be noticed that these waves were observed in the far tail of the comets, for example at distances $R>0.1$ AU down the tail for comet Kohoutek, whereas Rosetta did not venture out beyond $1000 \mathrm{~km}$.

Assuming that indeed there is a winding wave moving along the tail in the $X$-direction, which overtakes Rosetta when it is moving outward, with Rosetta moving at $v_{\mathrm{x}} \approx 1.3 \mathrm{~m} \mathrm{~s}^{-1}$, and passes over the spacecraft when Rosetta moves inward at $v_{\mathrm{x}} \approx 3 \mathrm{~m} \mathrm{~s}^{-1}$, a simple Doppler shift calculation can determine the speed of the wave, which results in $v_{\mathrm{h}} \approx 136 \mathrm{~m} \mathrm{~s}^{-1}$. Clearly, at a magnetic field strength of $\sim 10 \mathrm{nT}$, this velocity cannot represent the Alfvén velocity of the local plasma as in the helical waves discussed above.

On small scale physics in the tail the presence of the singing comet was investigated. It was shown that the power in the singing comet band $\left(10 \leq f_{\text {sing }} \leq 100 \mathrm{mHz}\right.$ ) was only strongly present during the outbound part and only for $R \leq 700 \mathrm{~km}$ and that it only reappeared during the inbound part of the tail excursion when $R<100 \mathrm{~km}$. The propagation direction of the waves in CSEQ coordinates, determined through MVA, varies in different intervals along the outbound part of the tail excursion, where the main component is in the $Z$-direction. As expected from the theory of the Weibel instability (Weibel 1959), assumed to be responsible for the singing comet (Meier et al. 2016), the propagation direction of the waves is at large angles $50^{\circ} \leq \alpha_{\mathrm{mvb}} \leq 100^{\circ}$ to the magnetic field. Interestingly, the propagation direction of the waves with respect to the radial direction to the comet, $\beta_{\mathrm{mvr}}$, also looks well behaved, also between $50^{\circ}$ and $100^{\circ}$. This probably means that the pick-up ions, generating the singing comet, are arriving from near radial direction, which should be verified by the RPC-ICA (Ion Composition Analyser; Nilsson et al. 2007 ) in an accompanying paper by Behar et al. (in prep.).

The instability creating the singing comet delivers energy into the magnetic field at a certain frequency $f_{\text {sing, which, to }}$ avoid an energy crises, has to dissipate through wave-particle interactions in a turbulent cascade. The power spectral density of the magnetic field oscillations was fitted with a power law, Eq. (3), between a lower frequency $f_{1}$, which is determined by the frequency at which the PSD is maximum in the singing comet frequency band, and a higher frequency $f_{1}$, which was set to $250 \mathrm{mHz}$. Only fits with $\mathcal{R}^{2}>0.95$ were taken into account and the results were split between singing dominated and no singing intervals. The spread in spectral index $\alpha$ was large in both, but there were different trends in either set, both for $\alpha$ as a function of radial distance $R$ from comet 67P and as a function of $P_{0}$.

The spectral index $\alpha$ as a function of radial distance $R$ showed that for the singing dominated intervals mainly $\alpha<-2$, out of 3007 points in Fig. 10A, 2188 points lie below -2 . For the no singing intervals at close distance to the comet, $R \leq 100$ $\mathrm{km}$, are polluted with the return of the singing and may pull the average $\alpha$ down. For distances $R>200 \mathrm{~km}$ the spectral index $\alpha>-2$, which is more towards the Kolmogorov range. This means that the presence of the singing comet, which includes a cross-field current for the Weibel instability (Weibel 1959; Meier et al. 2016), is influencing the turbulent cascade of energy.

For the spectral index $\alpha$ as a function of $\log \left(P_{0}\right)$ as shown in Fig. 10C and D the positive slope is explained by the fact that if there is more input at a certain frequency, $f_{1}$, more energy can cascade to higher frequency, and thereby reduce the steepness of the slope of the spectrum. The distribution of $\alpha$ for the singing dominated intervals $(\mathrm{C})$ is much flatter than that for the no singing intervals (D). Again, this seems to indicate that the presence of the cross-field current has an influence on the turbulent cascade.

The singing comet is limited to the region of radial distance $R \leq 700 \mathrm{~km}$, although on Leg 3, whilst Rosetta is hovering near $\sim 600 \mathrm{~km}$, there is no signal. This might be caused by two effects: first, the conditions for the Weibel instability are not adequate for significant wave growth; or, second, because of the deflection of the solar wind for the weakly outgassing comet, only one hemisphere is influenced by the cometary ions and the spacecraft is not in the right hemisphere at that time.

\section{Conclusions}

The interaction of the (very) weakly outgassing comet 67P with the solar wind does not lead to the usually expected bilobed induced magnetotail structure that has been observed in active comets such as 27P/Giacobini-Zinner. Because of the deflection of the solar wind, the cone-angle $\theta_{\mathrm{c}}$ in the tail region is rather large, indicating a more cross-tail-like field than a down-tail-like field. The clock-angle $\phi_{\mathrm{c}}$ of the magnetic field in the tail shows a rotation of the field, which is slowly moving down-tail.

The singing comet is present near the comet, $R \leq 700 \mathrm{~km}$, and has an influence on the turbulent cascade or the energy in the magnetic field. The average spectral index is larger for singing intervals than for quiet intervals.

The plasma physics around a weakly outgassing is only slowly beginning to be understood by the analysis of data from Rosetta RPC and new numerical modelling. This mission has opened a new window in space plasma physics.

Acknowledgements. The RPC-MAG data are available through ESA's Planetary Science Archive (PSA) and NASA's Planetary Data System (PDS). Rosetta is an ESA mission with contributions from its Member States and NASA. We acknowledge the staff of CDDP and IC for the use of AMDA and the RPC Quicklook database (provided by a collaboration between the Centre de Données de la Physique des Plasmas, supported by CNRS, CNES, Observatoire de Paris and Université Paul Sabatier, Toulouse and Imperial College London, supported by the UK Science and Technology Facilities Council). The work on RPC-MAG was financially supported by the German Ministerium für Wirtschaft und Energie and the Deutsches Zentrum für Luft- und Raumfahrt under contract 50QP 1401. We are indebted to the whole of the Rosetta Mission Team, SGS, and RMOC for their outstanding efforts in making this mission possible.

\section{References}

Acton, H. C. 1996, Planet. Space Sci., 44, 65

Alfvén, H. 1957, Tellus, 9, 92

Carr, C., Cupido, E., Lee, C. G. Y., et al. 2007, Space Sci. Rev., 128, 697

Edberg, N. J. T., Alho, M., André, M., et al. 2016, MNRAS, 462, S45

Ershkovich, A. I., \& Heller, A. B. 1977, Ap\&SS, 48, 365

Galeev, A. A., Cravens, T. E., \& Gombosi, T. I. 1985, ApJ, 289, 807

Glassmeier, K.-H. 2017, Phil. Trans. R. Soc. A, 375, 2016-0256

Glassmeier, K.-H., Boehnhardt, H., Koschny, D., Kührt, E., \& Richter, I. 2007a, Space Sci. Rev., 128, 1 
Glassmeier, K.-H., Richter, I., Diedrich, A., et al. 2007b, Space Sci. Rev., 128,649

Goetz, C., Koenders, C., Hansen, K. C., et al. 2016a, MNRAS, 462, S459

Goetz, C., Koenders, C., Richter, I., et al. 2016b, A\&A, 588, A24

Goetz, C., Volwerk, M., Richter, I., \& Glassmeier, K.-H. 2017, MNRAS, in press

Hansen, K. C., Altwegg, K., Berthelier, J.-J., et al. 2016, MNRAS, 462, S491

Hyder, C. L., Brandt, J. C., \& Roosen, R. G. 1974, Icarus, 23, 601

Ip, W.-H., \& Mendis, D. A. 1975, Icarus, 26, 457

Koenders, C., Glassmeier, K.-H., Richter, I., Ranocha, H., \& Motschmann, U. 2015, Planet. Space Sci., 105, 101

Koenders, C., Götz, C., Richter, I., Motschmann, U., \& Glassmeier, K.-H. 2016, MNRAS, 462, S235

Kolmogorov, A. N. 1941, Dokl. Akad. Nauk SSSR 32, 16

Mandt, K. E., Eriksson, A., Edberg, N. J. T., et al. 2016, MNRAS, 462, S9

Meier, P., Glassmeier, K.-H., \& Motschmann, U. 2016, Ann. Geophys., 34, 691

Nilsson, H., Lundin, R., Lundin, K., et al. 2007, Space Sci. Rev., 128, 671

Opitz, A., Karrer, R., Wurz, P., et al. 2009, Sol. Phys., 256, 365

Opitz, A., Fedorov, A., Wurz, P., et al. 2010, Sol. Phys., 264, 377

Plaschke, F., Goetz, C., Volwerk, M., et al. 2017, MNRAS, 469, S675
Raeder, J., Neubauer, F. M., Ness, N. F., \& Burlaga, L. F. 1987, A\&A, 187, 61 Richter, I., Koenders, C., Auster, H.-U., et al. 2015, Ann. Geophys., 33, 1031 Richter, I., Auster, H., Berghofer, G., et al. 2016, Ann. Geophys., 34, 609 Riedler, W., Schwingenschuh, K., Yeroshenko, Y. E., Styashkin, V. A., \& Russell, C. T. 1986 , Nature, 321,288

Schekochihin, A. A. 2017, J. Plasma Phys., submitted

Slavin, J. A., Smith, E. J., Tsurutani, B. T., et al. 1986, Geophys. Res. Lett., 13,283

Sonnerup, B. U. Ö., \& Scheible, M. 1998, in Analysis Methods for MultiSpacecraft Data, eds. G. Paschmann, \& P. Daly (Noordwijk: ESA), 185

Tao, C., Kataoko, R., Fukunishi, H., Takahashi, Y., \& Yokokama, T. 2005, J. Geophys. Res., 110, A11208

Volwerk, M., Vörös, Z., Baumjohann, W., et al. 2004, Ann. Geophys., 22, 2525

Volwerk, M., Jones, G. H., Broiles, T., et al. 2017, J. Geophys. Res., 122, 3308

Vörös, Z., Baumjohann, W., Nakamura, R., et al. 2004, J. Geophys. Res., 109, A11215

Weibel, E. S. 1959, Phys. Rev. Lett., 2, 83

Wicks, R. T., Horbury, T. S., Chen, C. H. K., \& Schekochihin, A. A. 2010, MNRAS, 407, L31 